\title{
GLIOMAS MÚLTIPLOS
}

\section{CASOS ILUSTRATIVOS DE QUATRO FORMAS DE APRESENTAÇÃO}

\author{
CLÉLIA MARIA RIBEIRO FRANCO*, SUZANA MARIA FLEURY MALHEIROS*, \\ ROBERTO GOMES NOGUEIRA **, MARCUS AZZAR SABRY BATISTA*, \\ ADRIALDO JOSÉ SANTOS*, NITAMAR ABDALA**, JOÃO NORBERTO STÁVALE***, \\ FERNANDO ANTONNIO PATRIANI FERRAZ****, ALBERTO ALAIN GABBAI*
}

\begin{abstract}
RESUMO - Os gliomas múltiplos são relativamente raros e podem ser classificados didaticamente de acordo com: a) a época da apresentação, em precoces (quando presentes desde o diagnóstico inicial) ou tardios (quando presentes durante a evolução); e b) as características dos exames de imagem, em multifocais (quando há evidência de contiguidade das lesões) ou multicêntricos (quando não é possível identificar contiguidade das lesões). Entre os 212 pacientes com diagnóstico anatomopatológico de glioma, acompanhados prospectivamente no setor de neurooncologia de março/90 a setembro/99, 15 (7\%) apresentaram lesões múltiplas. Descrevemos 4 casos característicos de cada uma das possíveis apresentações: multicêntrico precoce, multicêntrico tardio, multifocal precoce e multifocal tardio, com ênfase nas características de imagem e possíveis diagnósticos diferenciais. O diagnóstico diferencial das lesões múltiplas no sistema nervoso central inclui doenças inflamatórias e infecciosas, além de metástases. A possibilidade de tratar-se de tumores de origem glial, entretanto, deve ser sempre lembrada, mesmo naqueles pacientes com diagnóstico de neoplasia sistêmica conhecida, conforme já descrito na literatura. O diagnóstico histológico se impõe, uma vez que as características de imagem não permitem diagnóstico de certeza.
\end{abstract}

PALAVRAS-CHAVE: glioma, múltiplo, multifocal, multicêntrico, metástase.

\section{Multiple gliomas: four different presentations}

ABSTRACT - Multiple gliomas are uncommon and may be classified according to: a) the time of presentation in early (at diagnosis) or late (during treatment); b) the characteristics of computed tomography or magnetic resonance imaging (CT/MRI) in multifocal (with evidence of spread) and multicentric (without evidence of spread). From 212 patients with histopathologic diagnosis of glioma evaluated from March/90 to September/99, 15 (7\%) had multiple lesions. We describe 4 patients: early multicentric, late multicentric, early multifocal and late multifocal, with emphasis on characteristics of CT/MRI and possible differential diagnosis. The differential diagnosis of multiple lesions in the central nervous system includes mainly infectious/inflammatory diseases and metastasis, however multiple gliomas should always be considered, even in patients with known systemic cancer, as described by others. Considering that CT/MRI features are not definite, the diagnosis should always be confirmed by histopathologic examination.

KEY WORDS: glioma, multiple, multifocal, multicentric, metastasis.

A presença de lesões cerebrais múltiplas pode sugerir vários diagnósticos diferenciais, incluindo os tumores de origem glial. Os gliomas múltiplos (GsMs) têm sido mais descritos nas

Escola Paulista de Medicina, Universidade Federal de São Paulo (EPM/UNIFESP): São Paulo, SP: *Disciplina de Neurologia, **Disciplina de Radiologia, ***Disciplina de Anatomia Patológica, ****Disciplina de Neurocirurgia. Aceite: 14-outubro-1999.

Dra. Clélia M. Ribeiro Franco - Disciplina de Neurologia, EPM/UNIFESP - Rua Botucatu 740 - 04023-900

São Paulo SP - Brasil - Fax 5511575 5240. E-mail: clelia@unisys.com.br, suzana@sun-nepi.epm.br 
últimas décadas, paralelamente ao aparente aumento geral na freqüência das neoplasias primárias do sistema nervoso central ${ }^{1,2}$. Sua freqüência, muito provavelmente subestimada, varia de 1 a10\% dos gliomas ${ }^{1,3-8}$.

Os GsMs são considerados multicêntricos quando: 1. não houver contiguidade entre as lesões, seja macro ou microscópica; 2. as lesões não puderem ser consideradas como lesões satélites de um tumor primário; e 3. uma via determinada de disseminação, parenquimatosa ou liquórica, puder ser razoavelmente excluída. Quando esses requisitos não forem preenchidos, os gliomas são considerados multifocais $^{3,9}$. Alguns autores, porém, usam os termos multifocal e múltiplo com conotações superponíveis ${ }^{1,10,11}$. Kyritsis et al. ${ }^{1}$, consideram sem valor prático distinguir-se entre multicentricidade e multiplicidade/multifocalidade, preferindo defini-los apenas como gliomas multifocais precoces e tardios, dependendo da apresentação, seja desde o início do quadro, seja na evolução, durante ou após tratamento ${ }^{4,9,10}$. Esta classificação corresponde, de certa forma, aos casos anteriormente descritos como GsMs sincrônicos e metacrônicos ${ }^{6}$, ou seja, de apresentação simultânea no momento do diagnóstico ou não. Embora haja controvérsias, Jomin et al. ${ }^{10}$ sugerem que GsMs precoces e GsMs tardios tenderiam a graus de malignidade e comportamento diferentes, enfatizando a importância desta classificação.

Entre 212 pacientes com tumores gliais encaminhados ao Serviço de Neurologia da EPM, no período de março de 1990 a setembro de 1999, 15 (7\%) apresentaram lesões múltiplas pelos exames de imagem, seja na apresentação inicial ou na evolução, durante o tratamento. Destes 15 pacientes com gliomas múltiplos, 2 deles abandonaram o seguimento no Serviço. Dos 13 restantes, 9 tiveram diagnóstico de glioblastoma multiforme (GBM), 2 astrocitomas grau II e 2 astrocitomas anaplásicos. Apenas 4 deles tiveram apresentação precoce e a sobrevida mediana foi de 85 semanas (variação 5 a 182 semanas).

Apresentamos quatro dos pacientes que exemplificam as 4 possibilidades de apresentação clássicas dos GsMs: multifocal ou multicêntricos, precoces ou tardios.

\section{CASOS}

Caso 1 (multicêntrico precoce). NEC, 52 anos, sexo feminino. Três meses antes da admissão, passou a apresentar déficit de memória recente, desorientação têmporo-espacial, apatia e instabilidade emocional. A ressonância magnética (RM) na seqüência T1 com contraste mostrou duas lesões tumorais irregulares e infiltrativas, nas regiões temporais - uma maior à direita e uma de pequenas dimensões e mais posterior à esquerda. Devido à hipótese de metástase cerebral, foi realizada biópsia cerebral estereotáxica, com diagnóstico anatomopatológico de astrocitoma fibrilar (grau II da OMS), com componente gemistocítico. A família recusou qualquer tratamento, e a paciente faleceu 16 meses após o diagnóstico (Fig1A e B).

Caso 2 (multicêntrico tardio): MFNF, 39 anos, sexo feminino. A paciente apresentou hemiparesia direita, de instalação progressiva. Na época, tomografia computadorizada (TC) mostrou lesões tumorais com centro necrótico na região frontal esquerda (Fig 1C). Inicialmente a paciente recusou tratamento cirúrgico, retornando seis meses após início dos sintomas, com piora do déficit motor e crises parciais motoras à direita. Submetida a cirurgia, foi realizada ressecção parcial do tumor com diagnóstico anatomopatológico de GBM. Iniciou radioterapia (RT) e quimioterapia (QT) (BCNU $200 \mathrm{mg} / \mathrm{m}^{2}$ a cada 6 semanas), porém, 5 meses após o início da RT, em vigência de QT, apresentou hemiparesia progressiva à esquerda. A TC nessa fase mostrou lesão tumoral com centro necrótico na região frontal direita (Fig 1D). Reoperada, com ressecção completa do tumor, o diagnóstico anatomopatológico foi de GBM e radionecrose. Foi reiniciada QT (CCNU $110 \mathrm{mg} / \mathrm{m}^{2}$ no $1^{\circ} \mathrm{dia}+\mathrm{VCR} 1,4 \mathrm{mg} /$ $\mathrm{m}^{2}$ no $8^{\circ} \mathrm{e} 29^{\circ}$ dias, cada 8 semanas). Oito meses após, apresentou crise convulsiva generalizada tônico-clônica. A TC dessa época mostrou lesão tumoral com centro necrótico de grandes dimensões na região parietal esquerda, posterior ao local da lesão inicial, além de outra lesão tumoral de pequenas dimensões na região parietal direita (Fig 1E). A paciente faleceu 20 meses após o diagnóstico inicial.

Caso 3 (multifocal precoce): NRR, 45 anos, masculino. Há 5 meses da admissão, passou a apresentar crises uncinadas e vegetativas, até 4 vezes por dia, além de cefaléia, previamente episódica, que se tornou diária, contínua, sem melhora com analgésicos. A TC mostrou lesões tumorais nas regiões temporal e talâmica direitas 


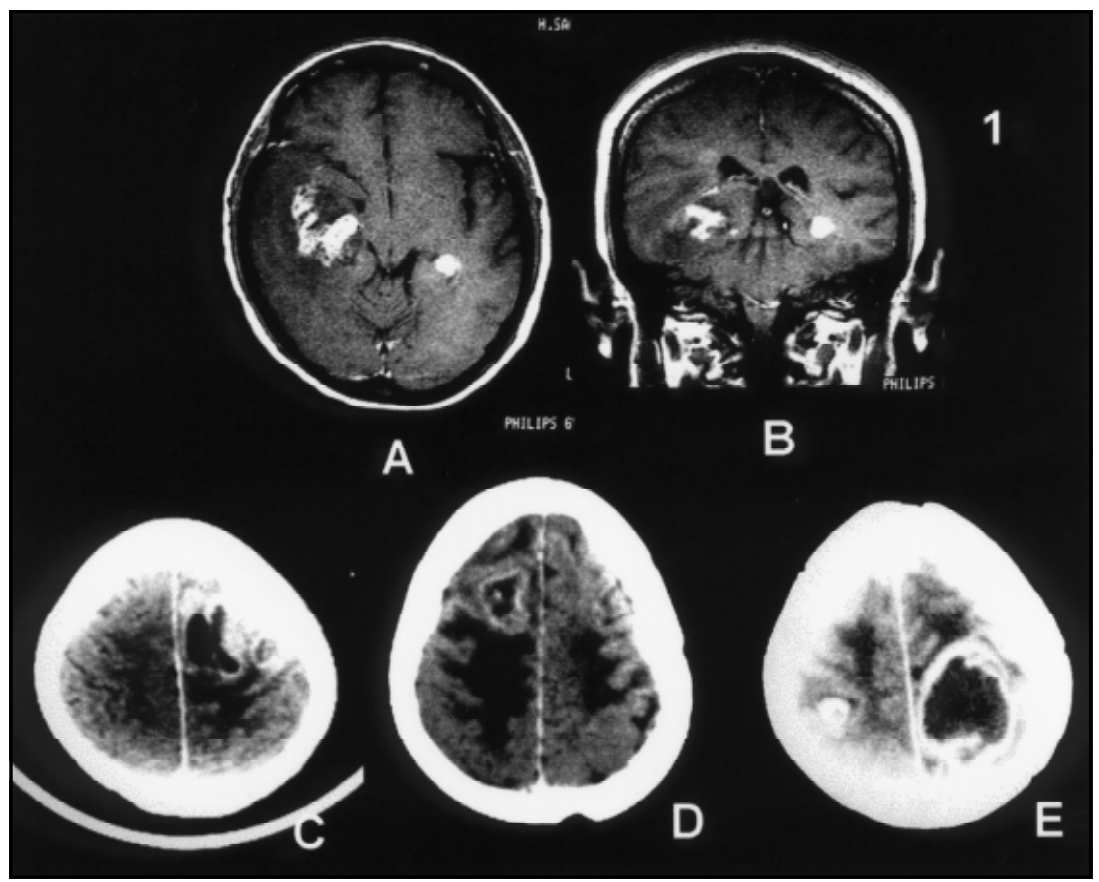

Fig 1. Gliomas multicêntricos precoce e tardio. (A) e (B): Caso 1, RM com lesões temporais bilaterais. (B), (C) e (D): Caso 2, TC inicial (B) com lesão frontal esquerda; TC 5 meses após início do tratamento $(C)$, com lesão frontal direita; e TC 8 meses após início do tratamento $(D)$, com lesões parietais bilaterais.

e no corno frontal esquerdo (Fig 2 A e B). O paciente chegou a receber tratamento de prova para toxoplasmose sem melhora. Submetido a biópsia estereotáxica, o diagnóstico anatomopatológico foi de GBM. Após ressecção parcial do tumor obteve melhora da cefaléia e controle das crises epilépticas com uso de dexametasona e fenitoína, sendo iniciada RT e QT (BCNU $200 \mathrm{mg} / \mathrm{m}^{2}$ ), quando evoluiu com crises parciais diárias e piora clínica progressiva. A TC na época mostrou lesões tumorais no lobo temporal direito, no tálamo com extensão para o átrio direito e no interior do corno frontal esquerdo, com extensão para o corno frontal direito e joelho do corpo caloso (fig. 2 C, D e E). Faleceu 12 meses após o diagnóstico.

Caso 4 (multifocal tardio): MGF, 25 anos, sexo feminino. Paciente com cefaléia contínua e intensa, com início 2 meses antes da admissão, seguida de diplopia e estrabismo convergente. A RM dessa época mostrou lesão tumoral talâmica esquerda sem áreas de necrose ou realces (fig. 3 A e B). Foi submetida a neurocirurgia, com ressecção parcial do tumor e diagnóstico anatomopatológico de astrocitoma fibrilar (grau II da OMS). Após 20 sessões de RT, o tratamento foi suspenso, devido à gravidez da paciente, que abandonou o acompanhamento. Seis meses após, retornou com quadro de hipertensão intracraniana. A TC dessa época mostrou lesão cicatricial talâmica esquerda e lesões tumorais nas regiões frontal e atrial do ventrículo lateral direito (Fig 3C, D e E). A paciente foi reoperada, com ressecção parcial do tumor e diagnóstico anatomopatológico de GBM. A sobrevida foi de 24 meses, a partir do diagnóstico inicial.

\section{DISCUSSÃO}

Os quatro pacientes descritos ilustram as possíveis formas de apresentação, de acordo com as diferentes classificações propostas: multicêntrico precoce no primeiro caso, multicêntrico tardio no segundo, multifocal precoce no terceiro e multifocal tardio no quarto caso.

A freqüência de GsMs varia de 1 até cerca de $10 \%$ dos gliomas ${ }^{1,3-6,8}$. Considerando-se apenas glioblastomas multiformes, esta frequiência pode elevar-se até $20 \%{ }^{12}$. A avaliação da frequiência 


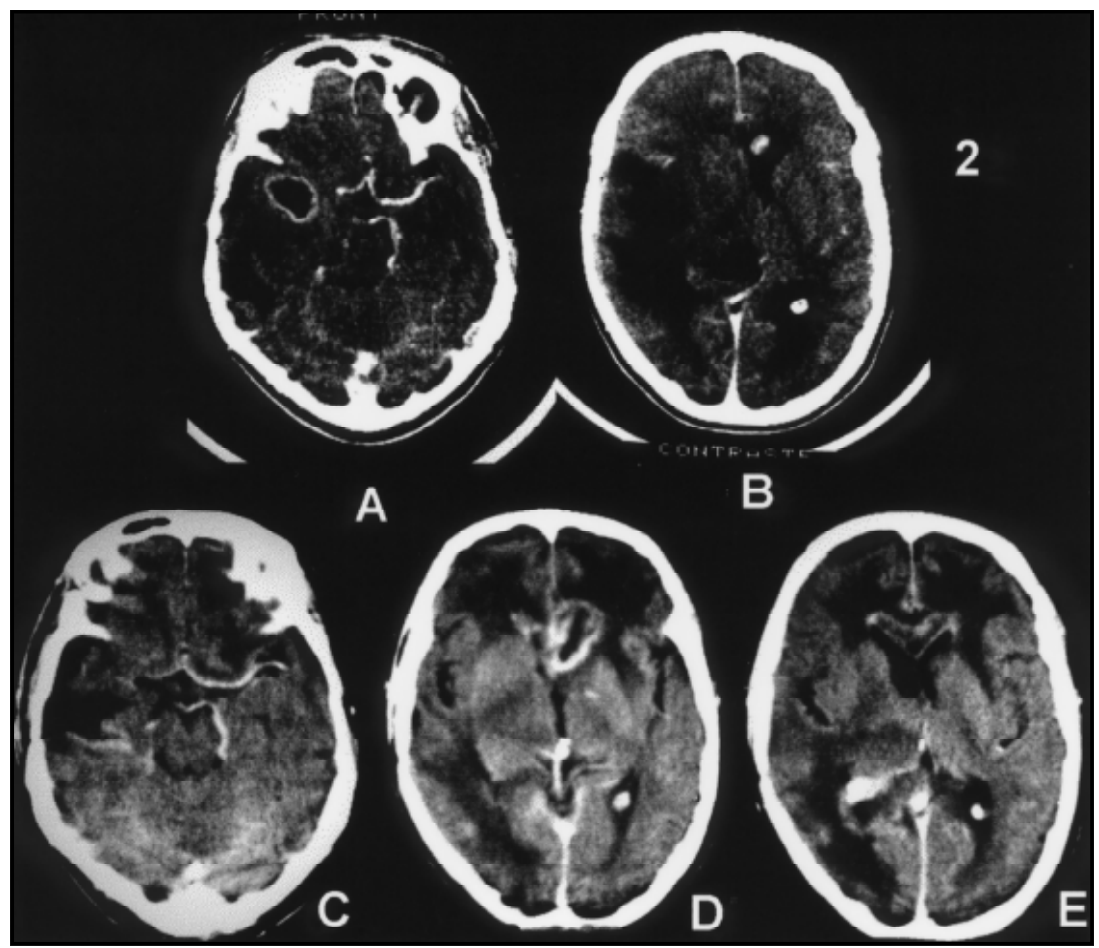

Fig 2. Glioma multifocal precoce. Caso 3. (A) e (B): TC com lesões tumorais nas regiões temporal e talâmica direitas, e corno frontal esquerdo. $(C),(D)$ e (E): TC após tratamento com RT e QT, com lesões tumorais temporal direita, átrio direito, corno frontal esquerdo, com extensão para corno frontal direito e joelho do corpo caloso.

real é dificultada pela variabilidade de métodos utilizados nos diferentes estudos. Esta freqüência é provavelmente subestimada ${ }^{4,12}$, considerando-se, por exemplo, o uso de diferentes critérios de seleção e de exclusão, diferentes critérios de definição de multiplicidade ou multicentricidade, e, principalmente, do método diagnóstico utilizado, seja por imagem ou necrópsia ${ }^{4,5,9,13}$. Mesmo nos estudos baseados em necrópsias, o exame macroscópico sistemático ou não do encéfalo, bem como o número de amostras examinadas, pode interferir nos resultados obtidos uma vez que a contiguidade entre as lesões pode ser subestimada ${ }^{4}$. Há ainda a possibilidade de que tumores aparentemente únicos ao diagnóstico representem a coalescência de focos tumorais múltiplos. Esta hipótese poderia explicar a ocorrência de quadros histolopatológicos diferentes em extremidades diferentes de uma mesma lesão ${ }^{4}$.

A idade de apresentação dos GsMs costuma variar entre a terceira e a sétima décadas ${ }^{1,3,4}$. Idade e sexo aparentemente não parecem diferir entre gliomas únicos ou múltiplos ${ }^{3}$.

Antes do advento da TC e do mais amplo acesso à biópsia cerebral, o diagnóstico se restringia a casos de necrópsia ${ }^{3,9}$. Atualmente, o uso de TC e RM permite melhor identificação das lesões múltiplas, tornando-se fundamental, no entanto, considerar as limitações destes métodos em relação à sensibilidade, quanto ao número e extensão das lesões, e especificidade, quanto à natureza das lesões ${ }^{1,6,8,14}$. Kyritsis et al. ${ }^{1}$ referem que a RM identificou melhor os limites das lesões e suas vias de disseminação, quando comparada à TC de crânio. $\mathrm{O}$ aparente aumento observado na frequiência dos GsMs nos últimos anos pode decorrer, em parte, da maior freqüência do diagnóstico com a introdução da TC e RM ${ }^{1,2}$, bem como do aumento de neoplasias do sistema nervoso central (SNC) em geral ${ }^{15}$, 


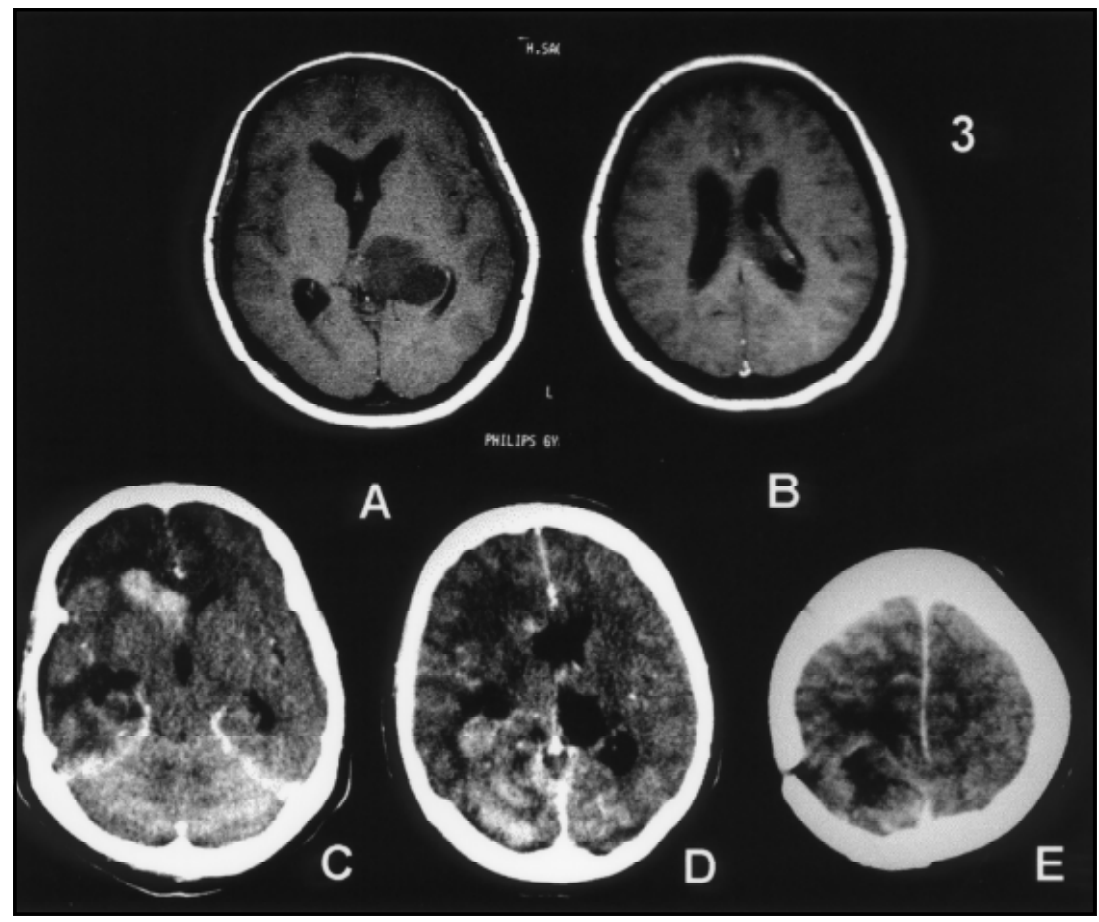

Fig 3. Glioma multifocal tardio. Caso 4. A e B: RM com lesão tumoral talâmica esquerda. (C), (D) $e(E)$ : TC com lesões tumorais frontal e atrial do ventrículo lateral direito.

além da maior possibilidade do diagnóstico histológico ${ }^{1}$, em função da morbidade e mortalidade mínimas de biópsias estereotáxicas atuais.

A localização mais relatada dos GsMs é a supratentorial, sem predileção hemisférica ${ }^{1,14}$, podendo ser, mais raramente, bilateral $^{6}$. Eles ocorrem mais comumente nos lobos frontais e parietais, seguidos pelos temporais, sendo raros nos lobos occipitais ${ }^{1,6}$. A grande maioria dos pacientes apresenta 2 ou 3 lesões, havendo descrições de até 15 lesões ${ }^{1}$. É rara a concomitância de lesões supra e infratentoriais ${ }^{16,17}$ bem como o acometimento cerebelar bilateral ${ }^{3}$, assim como do tronco cerebral ou o acometimento medular e encefálico de forma multicêntrica ${ }^{1,5,18}$.

As células gliomatosas podem se disseminar no próprio SNC, como metástases, aninhandose em múltiplos sítios. As vias de disseminação relatadas são os espaços perivasculares de VirchowRobin, leptomeninge e corpo caloso ${ }^{1,3,4,9}$. Batzdorf \& Malamud $^{9}$ argumentam, entretanto, que a relativa raridade de GsMs, quando comparada à freqüência com que se encontra infiltração dos espaços liquóricos seria um indício contra uma participação importante desta via. Awad et al..$^{19}$ identificaram 13 entre 70 pacientes em tratamento com gliomas supratentoriais, com evidência inequívoca de disseminação liquórica, afirmando que este parece ser um evento tardio, freqüentemente subestimado. A disseminação via vasos sanguíneos é considerada improvável, devido à espessura das paredes arteriais, ao diâmetro crescente das veias e à direção de seu fluxo, o que dificulta a impactação de células metastáticas ${ }^{3}$. Além disso, os GsMs raramente apresentam disseminação de acordo com os territórios vasculares ${ }^{3}$. Outra possibilidade de disseminação dos GsMs seria o desenvolvimento de múltiplos focos primários em várias regiões do encéfalo simultaneamente ${ }^{3}$.

Os tipos histológicos predominantes nos GsMs são o glioblastoma multiforme (grau IV da OMS) e o astrocitoma anaplásico (grau III da OMS) ${ }^{1,3,6}$, embora gliomas de baixo grau, 
oligodendrogliomas e tumores mistos ${ }^{4,17}$ e até associações de outros tumores sejam também relatadas $^{3,20}$. Jomin et al $(1983)^{10}$ sugerem que gliomas multicêntricos sejam neoplasias de baixo grau e que gliomas múltiplos sejam de alto grau de malignidade. Um mesmo GM, porém, pode apresentar diferentes graus e tipos histológicos em áreas distintas da mesma lesão ${ }^{4}$. Em termos de diagnóstico unicamente histológico, os GsMs não apresentam características que os diferenciem das lesões únicas. A variação histológica entre lesões diferentes é comparável à encontrada entre regiões diferentes de um mesmo tumor ${ }^{9}$.

Entre os diagnósticos de lesões encefálicas múltiplas, geralmente o primeiro diagnóstico é de metástases, como no Caso 2. Tassel et al. ${ }^{6}$ apontam características como a localização profunda, localização fora da junção córtico-medular, nódulo sólido com ausência de necrose central, comprometimento do mesmo hemisfério sem lesão contralateral e irregularidade da forma, como sinais de imagem pouco sugestivas de metástases, favorecendo o diagnóstico de GsMs. Ozawa et al. ${ }^{14}$, estudando imagens de RM, confirmaram que os GsMs costumam apresentar-se fora da junção córtico-medular, profundamente na substância branca supratentorial. Além disso, estes mesmos autores observaram serem os gliomas relativamente maiores, com realce irregular em anel mais espesso ao contraste e edema mais extenso, quando comparados às metástases ${ }^{14}$. Kyritsis et al. ${ }^{1}$ acrescentam que a evidência de disseminação meníngea, subependimal ou intraventricular sugere GsMs, porém, não há características de imagem que seguramente sejam capazes de distinguir metástases de GsMs ${ }^{1,6,21}$. Kyritsis et al. ${ }^{7}$ encontraram história prévia de neoplasias sistêmicas em $29,1 \%$ dos seus pacientes com GsMs de apresentação precoce, ressaltando a necessidade e a importância de confirmação histológica em qualquer paciente com suspeita de metástases. Parece portanto recomendável o diagnóstico histológico de lesões cerebrais múltiplas mesmo em pacientes com tumor primário conhecido ${ }^{1,4,7,22}$.

Com o aparecimento da síndrome de imunodeficiência adquirida (SIDA), torna-se de suma importância o diagnóstico diferencial com lesões de origem infecciosa e/ou inflamatória, como por exemplo toxoplasmose (como no Caso 3), abscessos, granulomas por tuberculose ${ }^{23}$ ou fungos, além de neoplasias, como linfoma primário do SNC, comum entre esses pacientes. Até mesmo hematomas e aneurismas parcialmente trombosados ${ }^{24}$ podem ser confundidos com GsMs.

Em países com alta prevalência de doenças infecciosas, como no Brasil, é fundamental estabelecer o diagnóstico diferencial com granulomas tuberculosos. Brismar et al..$^{23}$ apontam as dificuldades diagnósticas de granulomas tuberculosos e sua semelhança tomográfica com GsMs. Muito raramente, alguns pacientes com GsMs podem apresentar quadro clínico e de imagem simulando outras doenças, como por exemplo, vasculite ${ }^{25}$ e eslerose múltipla ${ }^{26}$. Por fim, a observação de lesões múltiplas durante ou após o tratamento de gliomas, torna necessário o diagnóstico diferencial entre GsMs e radionecrose ${ }^{6}$, como nos Casos 2 e 4.

Ainda não há uma definição precisa quanto ao melhor tratamento para os GsMs. Antes da introdução da TC, a abordagem cirúrgica era prejudicada pelo diagnóstico pré-operatório impreciso. É provável que a resposta destes tumores varie ainda mais que a dos gliomas únicos, devido a multiplicidade de quadros histológicos possíveis. Hochberg e Pruitt ${ }^{13}$ comentam os riscos e benefícios comparando irradiação de encéfalo total e irradiação localizada em pacientes com gliomas multicêntricos. Algo que realça a importância do diagnóstico histopatológico em todo paciente com lesões encefálicas múltiplas é a necessidade de doses mais elevadas de RT para GsMs do que para o tratamento das metástases encefálicas ${ }^{6,27,28}$. A QT aparentemente não interfere na evolução destes pacientes, uma vez que Choucair et al. ${ }^{5}$ não encontraram menor incidência de segundas lesões em pacientes que receberam QT para tratamento adjuvante de GBM ou astrocitoma anaplásico.

O prognóstico de pacientes com GsMs parece sombrio9 ${ }^{9}$, com sobrevida média após o diagnóstico de cerca de 6 meses para o grupo GsMs precoce e de 8 meses para o grupo tardio, com um número maior de sobreviventes a longo prazo neste grupo ${ }^{1}$. O prognóstico parece variar de acordo com o número, localização e quadro histológico das lesões, bem como com o tratamento realizado ${ }^{3,5}$. 
Embora não se possa confirmar a importância da distinção entre multicentricidade e multifocalidade, especialmente em relação ao prognóstico e terapêutica, o diagnóstico de gliomas múltiplos deve fazer parte do diagnóstico diferencial nos pacientes com lesões encefálicas múltiplas, mesmo naqueles pacientes com neoplasia sistêmica conhecida ${ }^{7}$. A confirmação do diagnóstico deve ser baseada ainda no exame histológico, uma vez que, nenhum exame de imagem permite o diagnóstico definitivo ${ }^{8,14}$.

\section{REFERÊNCIAS}

1. Kyritsis AP, Yung WKA, Leeds NE, Bruner J, Gleason MJ, Levin VA. Multifocal cerebral gliomas associated with secondary malignancies. (letter). Lancet 1992;339:1229-1230.

2. Helseth A. The incidence of primary central nervous system neoplasms before and after computerized tomography availability. J Neurosurg 1995;83:999-1003.

3. Courville CB. Multiple primary tumors of the brain: review of the literature and report of twenty one cases. Am J Cancer 1936;26:703-731.

4. Barnard RO, Geddes JF. The incidence of multifocal cerebral gliomas: a histologic study of large hemisphere sections. Cancer 1987;60:1519-1531.

5. Choucair AK, Levin VA, Gutin PH, et al.. Development of multiple lesions during radiation therapy and chemotherapy in patients with gliomas. J Neurosurg 1986;65:654-658.

6. Tassel PV, Lee Y-Y, Bruner JM. Synchronous and metachronous malignant gliomas: CT findings. AJNR 1988;9:725-732.

7. Kyritsis AP, Levin VA, Yung WKA, Leeds NE. Imaging patterns of multifocal gliomas. Eur J Radiol 1993;16:163-170.

8. Djalilian HR, Shah MV, Hall WA. Radiographic incidence of multicentric malignant gliomas. Surg Neurol 1999;51:554-557.

9. Batzdorf U, Malamud N. The problem of multicentric gliomas. J Neurosurg 1963;20:122-136.

10. Jomin M, Lesoin F, Lozes G, Delandsheer JM, Biondi A, Krivosic I. Les gliomes a foyers multiples: à propos de 10 observations. Neurochirurgie 1983;29:411-416.

11. Russell DS, Rubinstein LJ. Pathology of tumours of the nervous system. London: E. Arnold, 1959:152-153.

12. Scherer HJ. Le problème des gliomes multiples. Ver Neurol (Paris) 1936;65:178-179.

13. Hochberg FH, Pruitt A. Assumptions in the radiotherapy of glioblastoma. Neurology 1980;30:907-911.

14. Ozawa Y, Machida T, Noda M, et al.. MRI findings of multiple malignant gliomas: differentiation from multiple metastatic brain tumors. Radiat Med 1998;16:69-74.

15. Radhakrishnan K, Mokri B, Parisi JE, et al. The trends in incidence of primary brain tumors in the population of Rochester, Minnesota. Ann Neurol 1995; 37:67-73.

16. Salvati M, Oppido PA, Artizzu S, Fiorenza F, Puzzilli F, Orlando EP. Multicentric gliomas: report of seven cases. Tumori 1991; 77:518-522.

17. Kotwica Z, Papierz W. Cerebral and cerebellar glial tumors in the same individual. Neurosurgery 1992;30:439-441.

18. Sehgal AD, Gardner WJ. Separate gliomas of spinal cord and cerebellum in an individual: report of a case. J Neurosurg 1964; 21:802-804.

19. Awad I, Bay JW, Rogers L. Leptomeningeal metastasis from supratentorial malignant gliomas. Neurosurgery 1986;19:247251.

20. Madonick MJ, Shapiro JH, Torack RM. Multiple diverse primary brain tumours: report of a case with review of literature. Neurology 1961;11:430-436.

21. Prather JL, Long JM, van Heertum R, Hardman J. Multicentric and isolated multifocal glioblastoma multiforme simulating metastatic disease. Br J Radiol 1975; 48:10-15.

22. Giraud P, Petiot P, Vighetto A, Aimard G. Les tumeurs multiples (viscérales et neurologiques). Presse Med 1995;24:1736-1738.

23. Brismar J, Hugosson C. Larsson SG, Lundstedt C and Nyman R. Imaging of tuberculosis III: tuberculosis as a mimicker of brain tumor. Acta Radiol 1996;37:496-505.

24. Kandalaft N, Diehl J, Neuwelt EA. Nonneoplastic intracranial lesions simulating neoplasms on computed tomographic scan: excelent sensitivity with limited specificity. JAMA 1982;248:2166-2168.

25. Herman C, Kupsky WJ, Rogers L, Duman R, Moore P. Leptomeningeal dissemination of malignant glioma simulating cerebral vasculitis: a case report with angiographic and pathological studies. Stroke 1995;26:2366-2370.

26. Chakrabortty S S, Nagashima T, Saitoh M, Hanada Y, Hiyama K, Tamaki N. Intracerebral ring-enhancing lesions in a patient with multiple sclerosis: a case report. Surg Neurol 1995; 43:591-594.

27. Patchell RA. Metastatic brain tumors. Neurol Clin 1995;13:919-925.

28. Wen PY, Fine HA, Black P, Shrieve DC, Alexander E III, Loeffler JS. High-grade astrocytomas. Neurol Clin 1995;13:884-888. 